\title{
Succinate dehydrogenase-deficient gastrointestinal stromal tumor: from diagnostic dilemma to novel personalised therapy in 2 case reports
}

\author{
Madhawa De Silva ${ }^{1}$, Sameer Rastogi ${ }^{2}$, David Chan ${ }^{1}$, Christopher Angel ${ }^{3}$, Owen Prall ${ }^{3}$, Anthony Gill ${ }^{4}$, \\ Alexander Guminski ${ }^{1}$ \\ ${ }^{1}$ Department of Medical Oncology, Royal North Shore Hospital, Sydney, New South Wales, Australia; ${ }^{2}$ Department of Medical Oncology, All India \\ Institute of Medical Sciences, New Delhi, India; ${ }^{3}$ Department of Anatomical Pathology, Peter MacCallum Cancer Centre, Melbourne, Australia; \\ ${ }^{4}$ Department of Anatomical Pathology, Royal North Shore Hospital, Sydney, New South Wales, Australia \\ Correspondence to: Madhawa De Silva. Department of Medical Oncology, Level 1, ASB Building, Royal North Shore Hospital, St Leonards, Sydney, \\ NSW 2065, Australia. Email: mudsta1@gmail.com.
}

\begin{abstract}
Succinate dehydrogenase (SDH)-deficient gastrointestinal stromal tumor (GIST) is a unique and distinctive subtype of gastric GIST. The literature on this subtype from developing countries is exceedingly sparse. Patients with SDH-deficient GIST often experience a lack or delay in genomic profiling, despite stereotypical clinicopathologic features, potentially resulting in sub-optimal management. SDH-deficient GISTs are highly syndromic, typically have more indolent behavior, a prognosis not predicted by size and mitotic rate, a tendency to lymph node metastases, and are insensitive to standard tyrosine kinase inhibitors (TKIs). We report two women with SDH-deficient GIST. In the first case, SDH deficiency was identified late due to lack of awareness and poor access to diagnostic facilities. The patient progressed through TKI therapy, but responded to temozolomide, which is under investigation in clinical trials. In the second case, SDH deficiency was identified at diagnosis, and the patient responded well to ${ }^{177}$ Lutetium peptide radionuclide receptor therapy (PRRT) after progressing through two lines of TKIs. We aim to highlight the need for more awareness and access to genomic diagnostic facilities for GIST patients, temozolomide as a novel therapy for SDH-deficient GIST, and the potential value of DOTATATE positron emission tomography (PET) and PRRT as a novel imaging modality and therapy for TKI insensitive GIST patients.
\end{abstract}

Keywords: Metastatic gastrointestinal stromal tumor (GIST); succinate dehydrogenase (SDH); temozolomide; peptide radionuclide receptor therapy (PRRT); case report

Submitted Jan 22, 2021. Accepted for publication May 06, 2021.

doi: $10.21037 /$ tcr-21-131

View this article at: http://dx.doi.org/10.21037/tcr-21-131

\section{Introduction}

Gastrointestinal stromal tumors (GISTs) are the most common mesenchymal neoplasms of the gastrointestinal tract and are thought to originate from the interstitial cells of Cajal. The majority (90\%) of GISTs are activated by gain of function mutations in one of two type-III tyrosine kinase receptors, C-KIT and PDGFRA. Wild type GISTs (WT
GISTs) are conventionally defined as tumors that lack gain of function mutations in KIT and PDGFRA (1). Recent studies have shown that WT GIST is a heterogeneous entity with multiple subtypes. Of these various subtypes, $\mathrm{SDH}$-deficient GIST is the most common $(1,2)$.

The succinate dehydrogenase (SDH) complex comprises of 4 subunit proteins ( $S D H A, S D H B, S D H C$ and $S D H D)$ $(1,3,4)$. It is involved in the mitochondrial Krebs cycle and

$\wedge$ ORCID: 0000-0002-9437-3857. 
electron transport chain. SDHA and SDHB are responsible for enzymatic activity, while SDHC and SDHD anchor the complex to the mitochondrial inner membrane (1). If any component is lacking, the whole complex becomes unstable resulting in degradation of the SDHB subunit (4). Immunohistochemistry (IHC) for $S D H B$ becomes negative whenever there is mutation/inactivation in any of $S D H A$, $S D H B, S D H C$ and $S D H D$, and is therefore used as a highly sensitive marker for any subunit mutation in SDH (4). It is thought that $\mathrm{SDH}$ deficiency leads to neoplasia through metabolic stress and up-regulated hypoxia inducible factor 1 (HIF1), with increased insulin-like growth factor signaling (IGF) (1).

SDH-deficient GISTs have distinct clinicopathologic and molecular profiles compared to other GISTs $(2,5)$. Briefly, patients with SDH-deficient GIST typically present at a young age, are more likely to be female, and have a tendency to lymph node metastasis (rare in other GISTs). With the exception of a single case report SDHdeficient GISTs exclusively arise from the stomach (6). There are some morphological clues to SDH-deficient GIST including a lobulated and multifocal growth pattern and frequent epithelioid histology, but only loss of IHC for $S D H B$ (so called 'SDH deficiency') is considered definitively diagnostic $(2,5)$. Deficiency of SDH in GIST can happen either from mutation in the genes encoding the SDH subunits, SDHA, SDHB, SDHC or SDHD (collectively referred to as $S D H x$ mutations) or through epigenetic mechanisms leading to silencing of $S D H C$ (inactivation through promoter hypermethylation) $(1,7,8)$.

SDH-deficient GIST can present as one of 2 classic syndromes, Carney triad and germline SDH mutation syndrome (sometimes termed 'Carney-Stratakis syndrome') $(1,7,8)$. Carney triad is a non-heritable syndrome characterised by multiple gastric GISTs, paragangliomas and pulmonary chondromas, and is attributable to postzygotic epigenetic SDH inactivation through $S D H C$ hypermethylation $(1,7,8)$. Approximately half of SDHdeficient GISTs are associated with $S D H C$ hypermethylation (Carney triad); $30 \%$ are associated with germline $S D H A$ mutation (in addition to loss of $S D H B$ expression these tumours also show loss of expression of SDHA by IHC) and the remaining $20 \%$ are associated with $S D H B, S D H C$ or $S D H D$ mutation $(3,4,9)$. Somatic only mutation in the SDH genes in the absence of germline mutation or SDHC epimutation is rare, but does occur (4).

Surgery remains a key intervention in the management of early GIST, with cure achieved in up to $60 \%$ with surgery alone in the pre-imatinib era (10). GISTs are typically resistant to traditional chemotherapy, however, the majority with oncogenic C-KIT or PDGFRA mutations are sensitive to oral tyrosine kinase inhibitors (TKIs), which are standard of care for advanced disease (11-13). In 2016, Patrikidou et al. reported long-term outcomes of metastatic GIST patients treated with standard dose imatinib in the French Sarcoma Group BFR14 study. Landmark survival was demonstrated with a median overall survival (OS) of 55 months, in a chemoresistant disease with objective response rates less than $5 \%$ and median overall survival around 20 months prior to imatinib (11,12). In 2017, Casili et al. reported updated 10-year survival of patients with metastatic GIST treated with imatinib at two doses. In this multicenter international randomized study of 946 patients with a median follow up time of 10.9 years, median OS was 47 months (13). There was no difference in survival for the higher dose arm, except for KIT exon 9 mutated patients with longer progression-free survival (PFS) at a higher dose (HR, 0.40; 95\% CI, 0.22 to 0.72 ) (13). Eventually, secondary resistance emerges through KIT mutations, for which there are limited systemic treatment options. Few studies have directly compared currently available post-imatinib second-line treatments, and several trials are recruiting. Compared to placebo, second and third-line treatment with sunitinib and regorafenib yield additional PFS benefits in the order of 5 and 4 months respectively (14-16). Interpretation of OS is limited in this setting due to small numbers, limited follow up, TKI as a comparator arm, and cross over, however, sequential use of TKIs will improve survival compared to time under imatinib alone. The recent development of novel molecular compounds is promising, and will enable more personalized therapy (17). Alternating TKIs, immunotherapy, and combination treatments are also being explored (17-19). Patients with WT GIST have primary resistance to TKIs due to up-regulation of alternate oncogenic pathways. There remains no high-impact drug therapy for these patients, who typically progress through various TKIs. Given the different biology and behavior of these tumors, a different approach to treatment is required.

In considering alternative therapies for patients with TKI refractory or wild-type disease, prior in vivo work has demonstrated that GIST tumours express somatostatin receptors and bind somatostatin analogues, and therefore may respond to peptide radionuclide receptor therapy (PRRT) (20-23). In 2013, Arne et al. demonstrated significant expression of SSTR1 and SSTR2, with low levels of SSTR3-5 in GIST tumours via quantitative 
PCR. Presence of protein was also confirmed via IHC. Also demonstrated was uptake of ${ }^{111}$ Indium-octreotide in three of six GIST patients, and in vivo binding plus internalisation of ${ }^{177}$ lutetium-octreotide (20). In 2014, Zhao et al. again demonstrated presence of SSTR1 and 2 in over $80 \%$ of 453 GIST samples by IHC (21). In this study, absence of SSTR2 was an independent prognostic marker, associated with reduced recurrence-free survival in the resected setting (21). In 2016, Paulmichl et al. performed incubation of ${ }^{68} \mathrm{Ga}$-DOTA-octreotate (targeting SSTR2) with imatinib sensitive and imatinib insensitive cell lines. Low levels of specific binding and internalisation were observed (23). With high expression of SSTRs in GISTs, new therapeutic strategies such as PRRT and somatostatin analogues hold promise, though pre-clinical studies have yielded conflicting results. To our knowledge, we present the first case report of a patient with metastatic GIST treated with PRRT following progression through TKI therapy.

There have been few reports of GIST patients from centers in India, and reported data on mutation testing (C-KIT/PDGFRA or SDH) is conspicuously absent $(24,25)$. Similarly, there is no report of SDH mutated GIST from India in the English literature. This reflects the prevailing lack of dedicated clinics for rare disease and lack of testing for rare diseases like GIST (26). We present a report of a young lady with SDH-deficient GIST from Nepal, who travelled to India for initial management, and though suspected to be SDH-deficient, this was not able to be confirmed due to lack of diagnostic facilities. Her tumour was later demonstrated to be SDH-deficient after moving to Australia. After progressing through TKIs, she had a response followed by disease stabilization with temozolomide, which is under investigation in this setting. We present the following article in accordance with the CARE reporting checklist (available at http://dx.doi. org/10.21037/tcr-21-131).

\section{Case presentation}

\section{Case 1}

In May 2012, a 23-year-old year old female with no prior medical history presented to her local health service in Nepal with incidental detection of an abdominal mass during review for upper respiratory tract infection. Contrast enhanced computed tomography (CT) revealed a $10 \mathrm{~cm} \times 8 \mathrm{~cm}$ mass in the greater curvature and body of the stomach, a mass in the lesser curvature, and several liver lesions suspicious for metastases. She was referred to a metropolitan hospital in India, where she underwent subtotal gastrectomy and gastrojejunostomy in October 2012. Histopathology revealed GIST, and she was started on first-line imatinib, with no molecular testing performed at that time.

After 5 years on imatinib, the patient had symptoms of progression and re-presented in September 2018. Repeat imaging revealed soft tissue density in the greater curvature suggestive of local recurrence, a $2 \mathrm{~cm}$ perigastric lymph node, and marginal progression in number and size of her liver metastases. C-KIT and PDGFRA mutation testing was performed in a private lab in India, revealing a PDGFRA exon 18 mutation. The patient was started on sunitinib in December 2018, and in January 2019 she moved to Australia. Two months later, she had an episode of GI bleeding for which she received symptomatic care including blood transfusion and palliative radiotherapy to the gastric mass (8 Gy, 1 fraction). Meanwhile she continued sunitinib.

In April 2019 (after 4 months of sunitinib), restaging revealed partial response in the gastric mass, but increasing liver metastases. C-KIT and PDGFRA mutation testing was repeated in Australia in a NATA accredited laboratory, and in contrast to the results found in the previous laboratory no PDGFRA (or KIT) mutation was identified. SDHB IHC was then performed in Australia, revealing SDH deficiency (Figure 1). Restaging in June 2019 demonstrated further progressive disease (Figure 2). At this point, the patient commenced temozolomide at $150 \mathrm{mg} / \mathrm{m}^{2}$ for 5 days, every 28 days. She tolerated this well, and the next cycle was given at $200 \mathrm{mg} / \mathrm{m}^{2}$. A progress fluorodeoxyglucose (FDG)-positron emission tomography (PET) scan after 4 cycles demonstrated stable disease, and a subsequent CT after 7 cycles (January 2020) revealed a partial response (Figure 3). The patient remains clinically well not requiring blood transfusions, and serial restaging with CT up to the last scan in December 2020 (after 18 cycles) shows stable disease since January 2020 (Figure 3). Genetic testing for germline SDH mutation has not been performed yet due to insufficient resources.

\section{Case 2}

The patient is a 60-year-old lady of Armenian heritage, born in Iran then migrating to Australia. She had a past medical history of hypertension and gout, and initially presented to a metropolitan hospital in Sydney in late 2017 with symptomatic anaemia and a palpable 


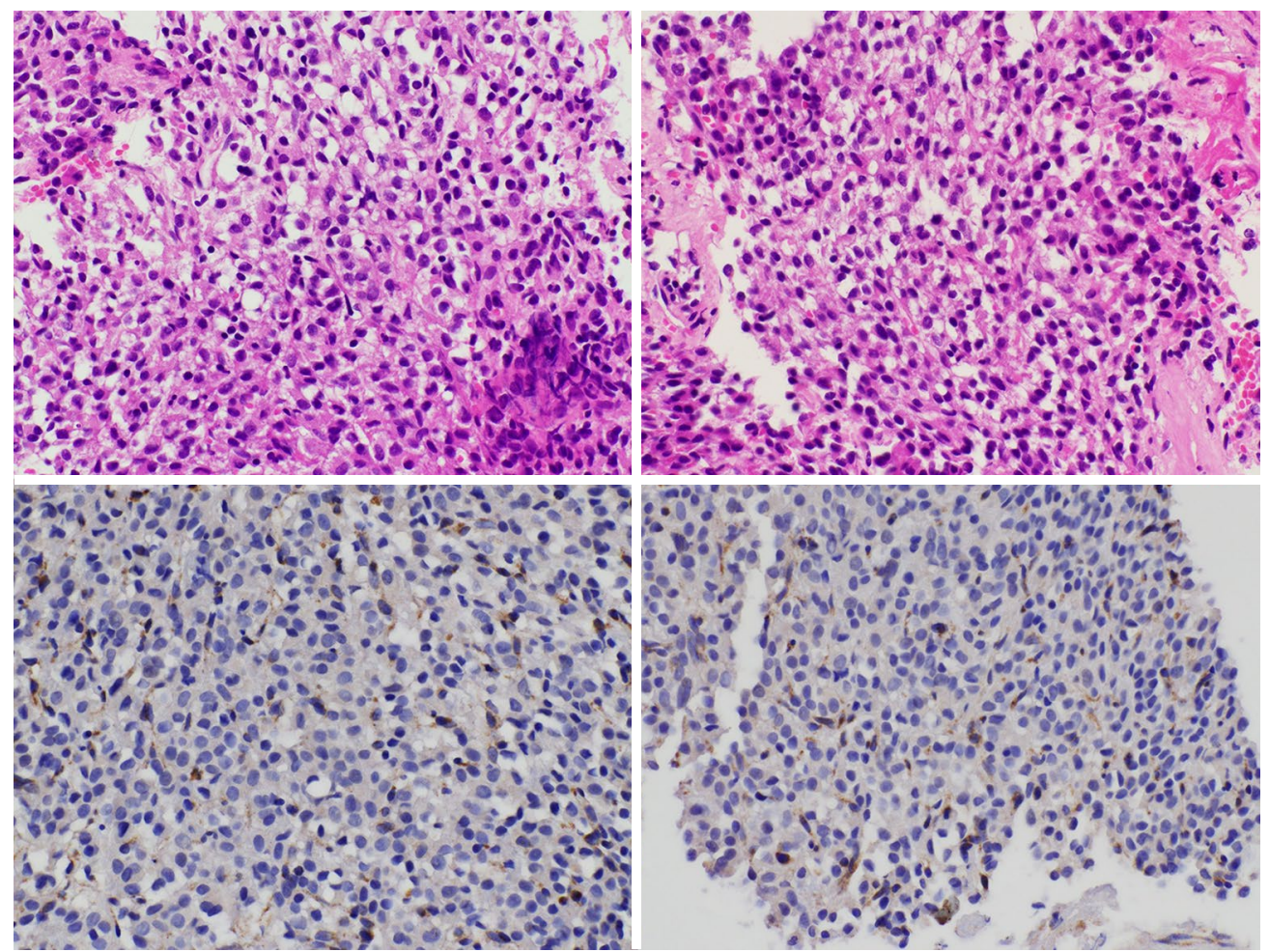

Figure 1 Hematoxylin and eosin (above) and immunohistochemically stained sections (below). SDHB staining is negative in the tumor cells (above), but positive in endothelial cells (below) which are a good internal control (original magnifications 400×).
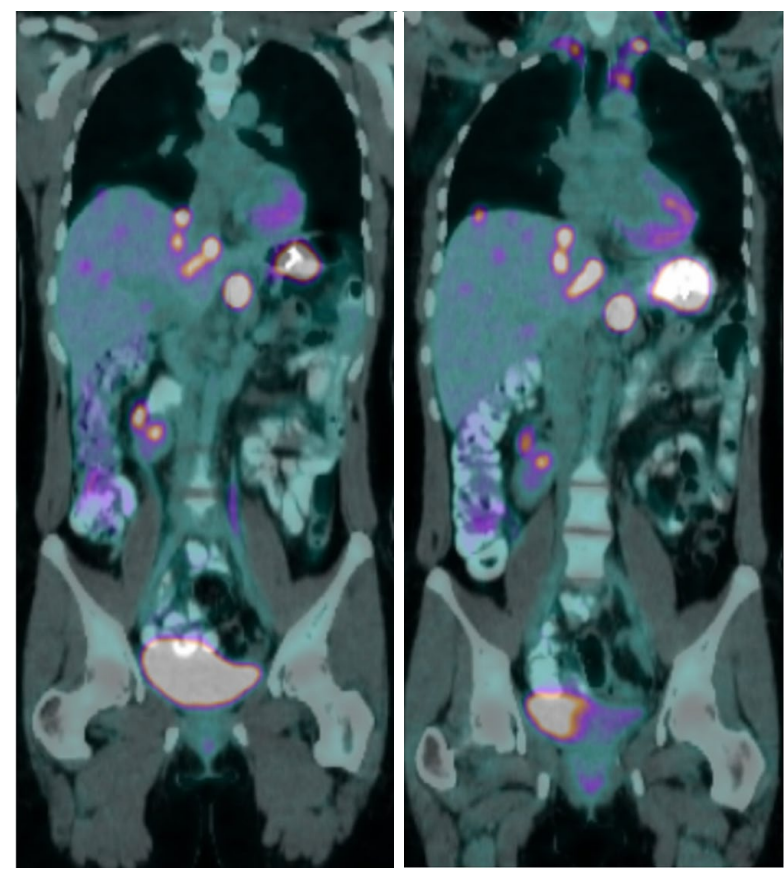

Figure 2 Restaging fluorodeoxyglucose positron emission tomography scans from January 2019 (Left) and June 2019 (Right) demonstrating disease progression on sunitinib, with increasing size and avidity of the posterior gastric wall tumour and liver metastases. The patient ceased sunitinib and commenced temozolomide in July 2019. 

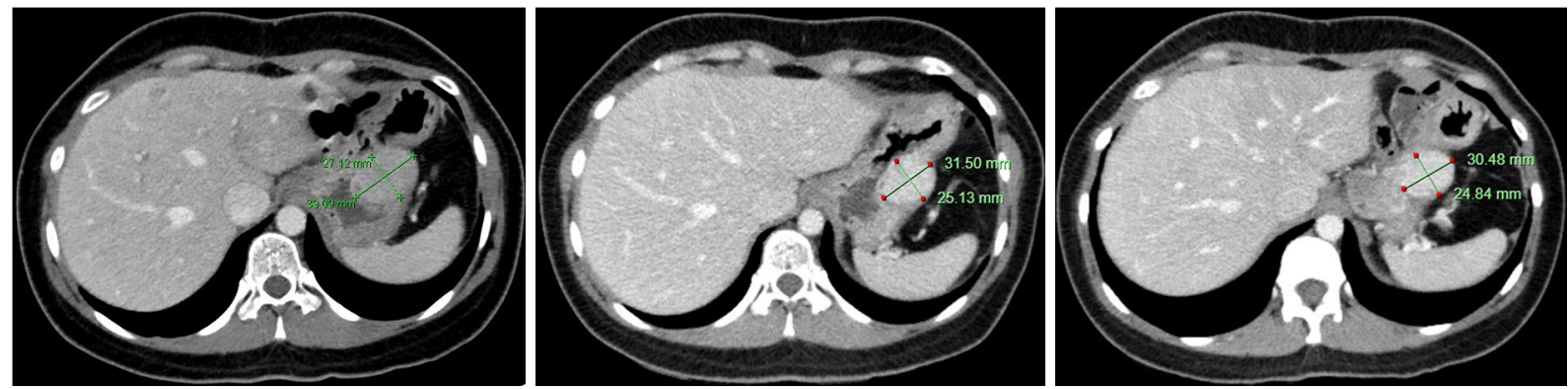

Figure 3 Restaging computed tomography scans from April 2019 (Left), January 2020 (Middle), and December 2020 (Right) demonstrating initial response to temozolomide followed by stabilization of disease.

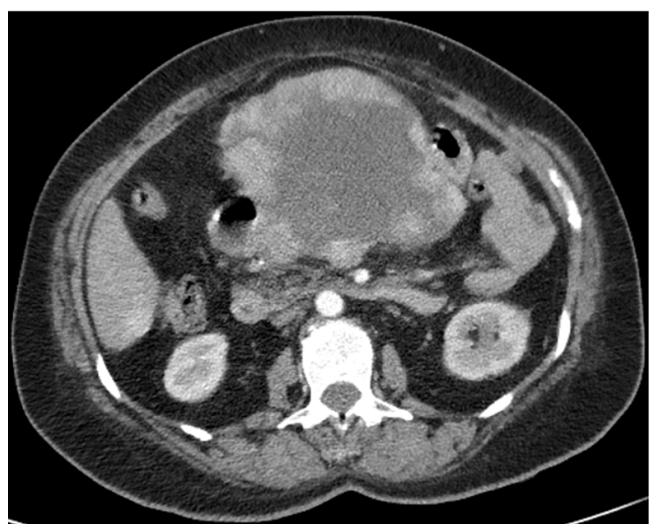

Figure 4 Initial computed tomography in late 2017 which revealed a large mass centered on the pancreatic neck, with liver, lung, and adrenal deposits.

epigastric mass. Blood tests revealed severe anaemia (hemoglobin 44) and iron deficiency. Contrast enhanced CT demonstrated a large lobulated lesion centered at the pancreatic neck displacing the liver and stomach, with bilateral adrenal, lung and liver deposits (Figure 4). Endoscopy revealed a large submucosal gastric mass with an ulcerated apex, but no active bleeding. Biopsy of this lesion was not performed due to the diagnostic endoscopic appearance of GIST. In hindsight, the patient had no other upper or lower gastrointestinal symptoms, and had been constitutionally well.

Biopsy of a liver metastases was subsequently performed. This confirmed metastatic GIST with a mitotic count of 2 per 50 high-power fields. IHC was positive for C-KIT and DOG1, but negative for both SDHA and SDHB (Figure 5).

The patient commenced imatinib in January 2018, and within one month, there was clinical and radiologic progression. She therefore commenced second-line sunitinib in February 2018, and underwent genetic testing which revealed novel pathogenic germline variant in $S D H A$ (SDHA c. $1226 \mathrm{~A}>\mathrm{T}$ ).

Restaging in June 2018 confirmed further disease progression. Due to the presence of adrenal nodules and a germline SDHA mutation, a ${ }^{68} \mathrm{Ga}$-DOTATATE PET scan was arranged to investigate intercurrent pheochromocytoma, paraganglioma or neuroendocrine neoplasm. This revealed DOTATATE-avid disease in the liver, abdominal lymph nodes, a small right pelvic bone metastasis, and uptake above the right $\mathrm{C} 1$ arch (Figure 6). Following multi-disciplinary discussion and an FDG-PET scan showing concordant disease (DOTATATE > FDG), the patient went on to receive ${ }^{177}$ lutetium-DOTA-octreotate PRRT in September 2018, eventually completing four cycles in May 2019.

PRRT was tolerated well, however there was delay due to myelosuppression. A progress ${ }^{68} \mathrm{Ga}$-DOTATATE PET scan in July 2019 showed a good partial response to PRRT, with decreased size of the primary mass and liver metastases, and overall stable avidity (Figure 7). The patient continued on observation, and a repeat ${ }^{68} \mathrm{Ga}$-DOTATATE PET in November 2019 revealed further reduction in the primary with necrotic change (Figure 8). Interval restaging with CT in February 2020 demonstrated ongoing response (Figure 9), however, repeat ${ }^{68} \mathrm{Ga}$-DOTATATE and FDG-PET scans in July 2020 revealed concordant disease progression in bone, liver, and lung (DOTATATE > FDG). Due to borderline renal function, the patient was not eligible for further PRRT, and therefore commenced temozolomide in September 2020. A progress ${ }^{68} \mathrm{Ga}$-DOTATATE PET in December 2020 revealed a mixed response (Figure 10). Importantly, there was a reduction in the burden of hepatic disease, with low volume extra-hepatic progression. 

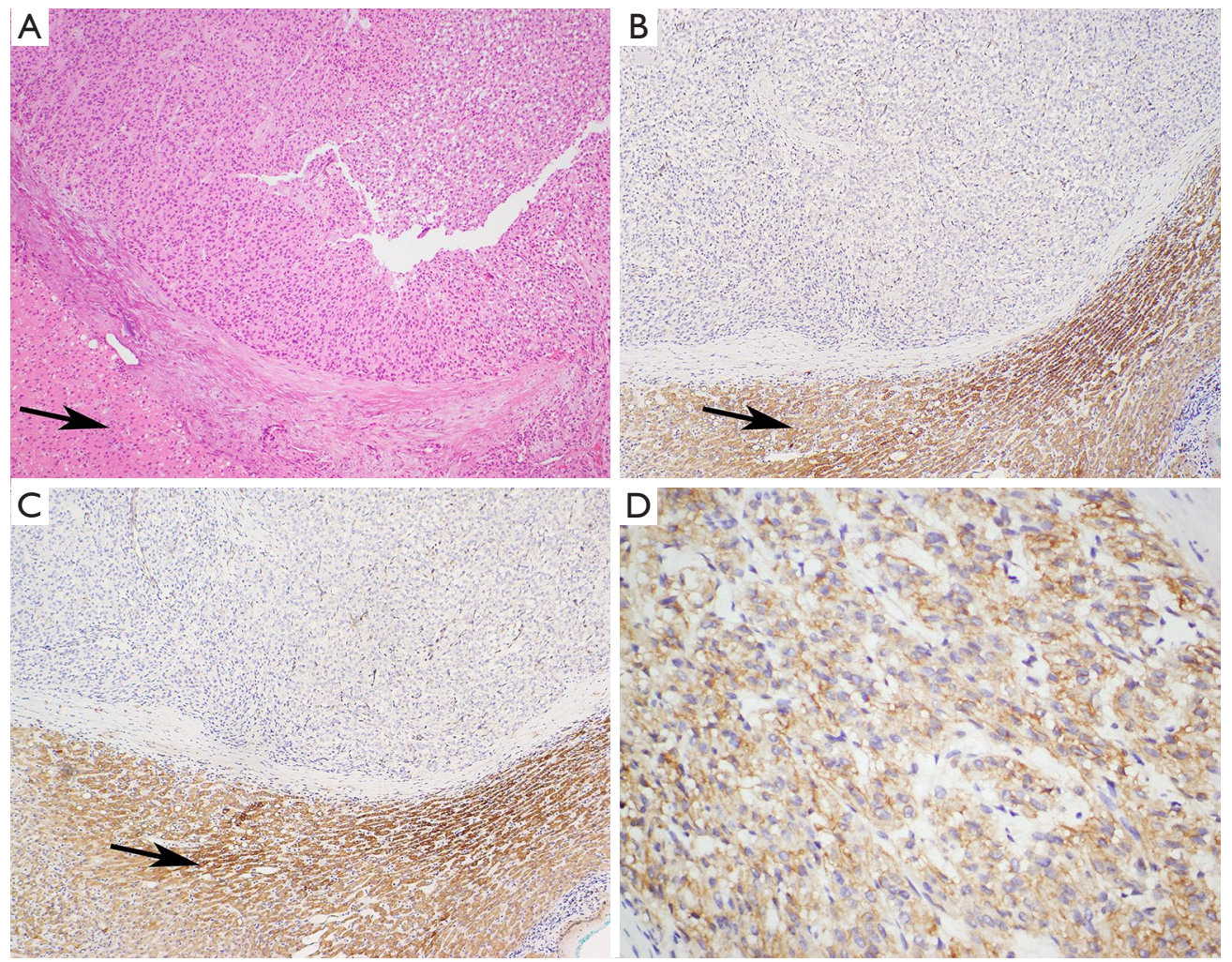

Figure 5 Serial Hematoxylin and eosin (H\&E) and immunohistochemically (IHC) stained sections from the liver metastasis. (A) The tumour demonstrated an epithelioid morphology - typical for SDH-deficient GIST. There was loss of immunohistochemical expression of both SDHB (B) and SDHA (C) with preserved positive staining in the non-neoplastic internal control (normal liver tissues, arrows). The loss of SDHB expression confirms the diagnosis of SDH-deficient GIST. The co-occurrence of SDHA loss indicates that the tumour is driven by biallelic SDHA mutation/deletion/inactivation which is almost always associated with a germline SDHA mutation (9). IHC for somatostatin receptor 2A (D) demonstrates diffuse strong positive expression $(27,28)$ (A H\&E, B SDHB IHC, C SDHA IHC, D SSTR2A IHC, original magnifications A-C 200×, D 400×).

The patient remains clinically well and continues on temozolomide with no major drug-related toxicities.

All procedures performed in studies involving human participants were in accordance with the ethical standards of the institutional and/or national research committee(s) and with the Helsinki Declaration (as revised in 2013). Written informed consent was obtained from the patients.

\section{Discussion}

Case 1 highlights the difficulty in diagnosis and management of rare tumors like SDH-deficient GIST in developing countries, however, lack of awareness and access to appropriate diagnostic facilities also occurs in nonmetropolitan areas of developed countries. The clinical presentation was suggestive of an SDH-deficient GIST, yet this was unknown for years, and the patient was started on imatinib inadvertently. The pace of progression was very much similar to that reported in literature for wild type GIST, and was unusually indolent (29). SDH-deficient GIST accounts for approximately $5 \%$ of gastric GISTs ( $3 \%$ of all GISTS) in a completely unselected population, but incidences of up to $7.5 \%$ to $10.8 \%$ of gastric GISTs have been reported in populations with a referral bias towards younger patients $(2,4,30)$. In one study, all patients with GIST less than 21 years of age were SDH-deficient while almost half of the patients aged 21 to 30 years were SDH-deficient, reflecting GIST in young patients should have high suspicion of SDH-deficiency (30). In the same series, non-gastric GIST patients were also tested for SDH deficiency $(n=378)$ but none were SDH-deficient, reinforcing the fact that these tumors are mostly limited to 

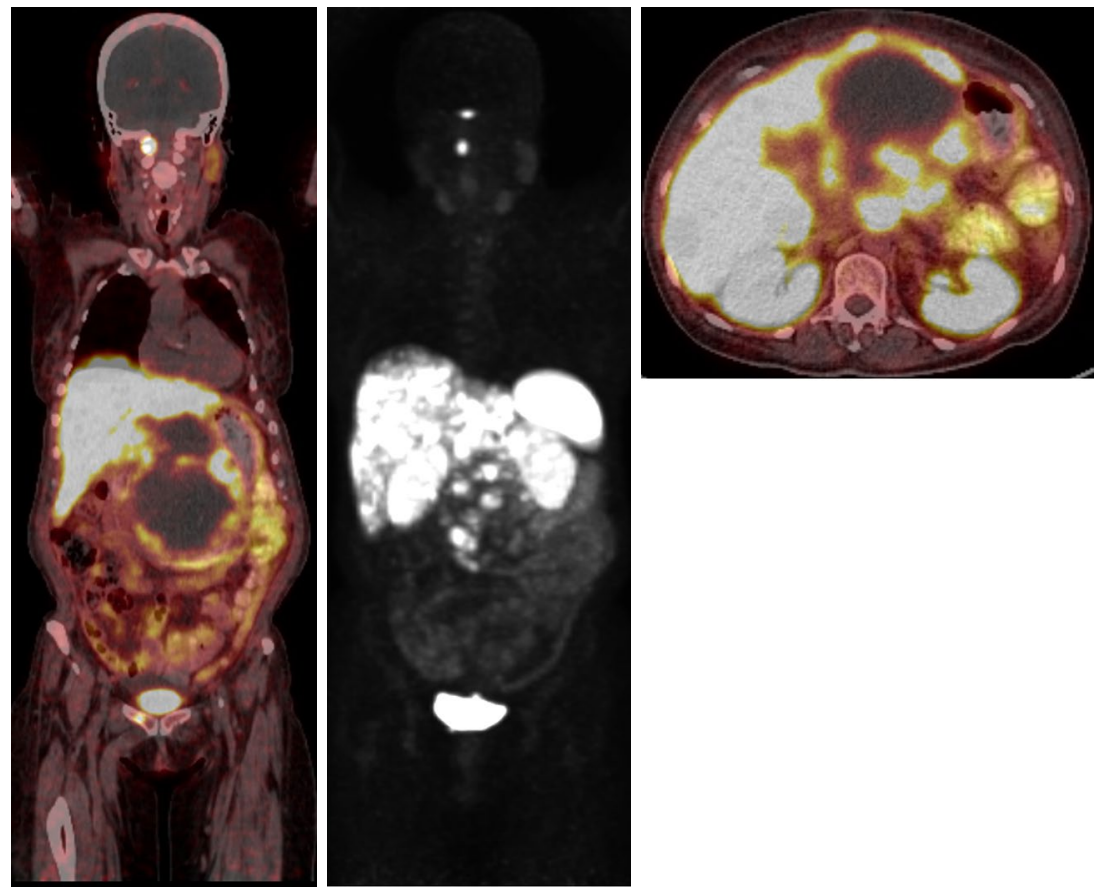

Figure 6 Initial ${ }^{68} \mathrm{Ga}$-DOTATATE positron emission tomography scan performed in July 2018 demonstrating peripheral DOTATATE uptake in the large central abdominal mass with a necrotic core. DOTATATE uptake was also seen in intra-abdominal lymph nodes, liver metastases, bilateral adrenal nodules, right pubic body, and above the right first cervical vertebral arch.
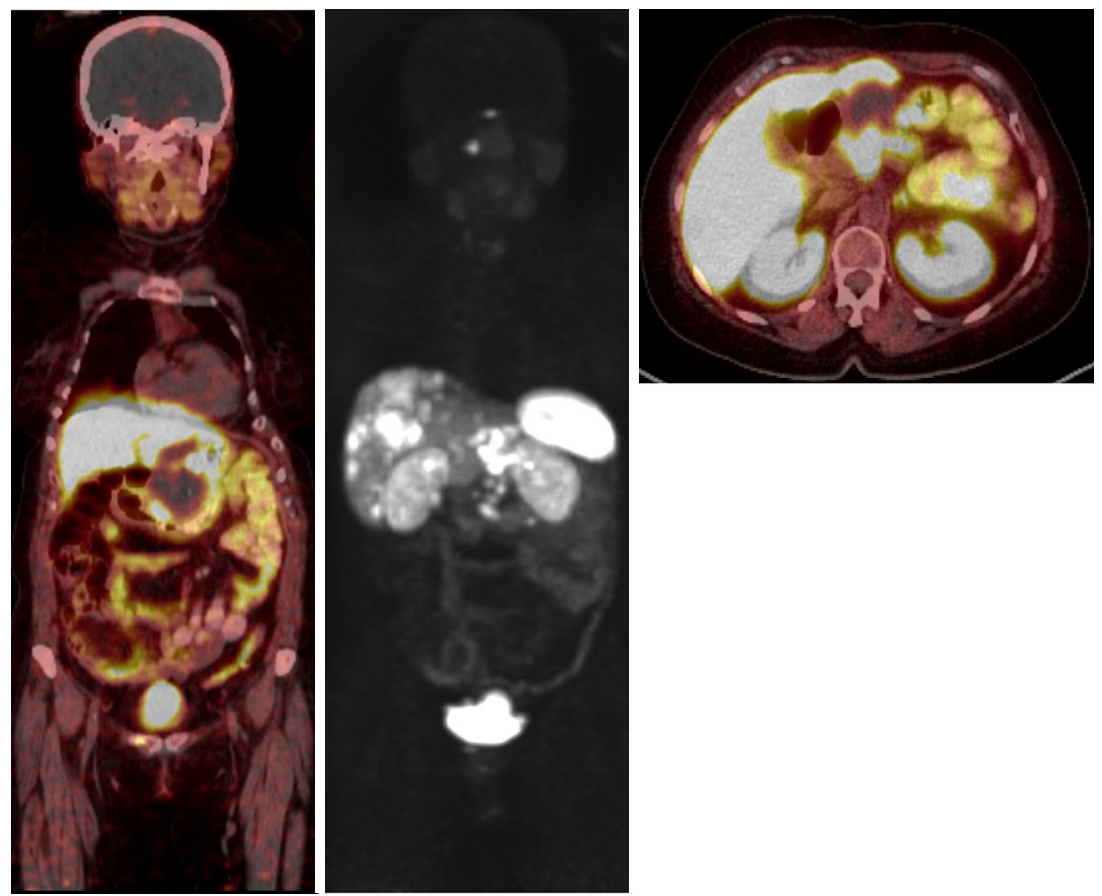

Figure 7 Restaging ${ }^{68}$ Ga-DOTATATE positron emission tomography scan performed in July 2019 after completing 4 cycles of ${ }^{177}$ Lutetium peptide radionuclide receptor therapy demonstrating partial response. There was a reduction in the primary mass, liver and nodal disease, with overall stable DOTATATE uptake. 

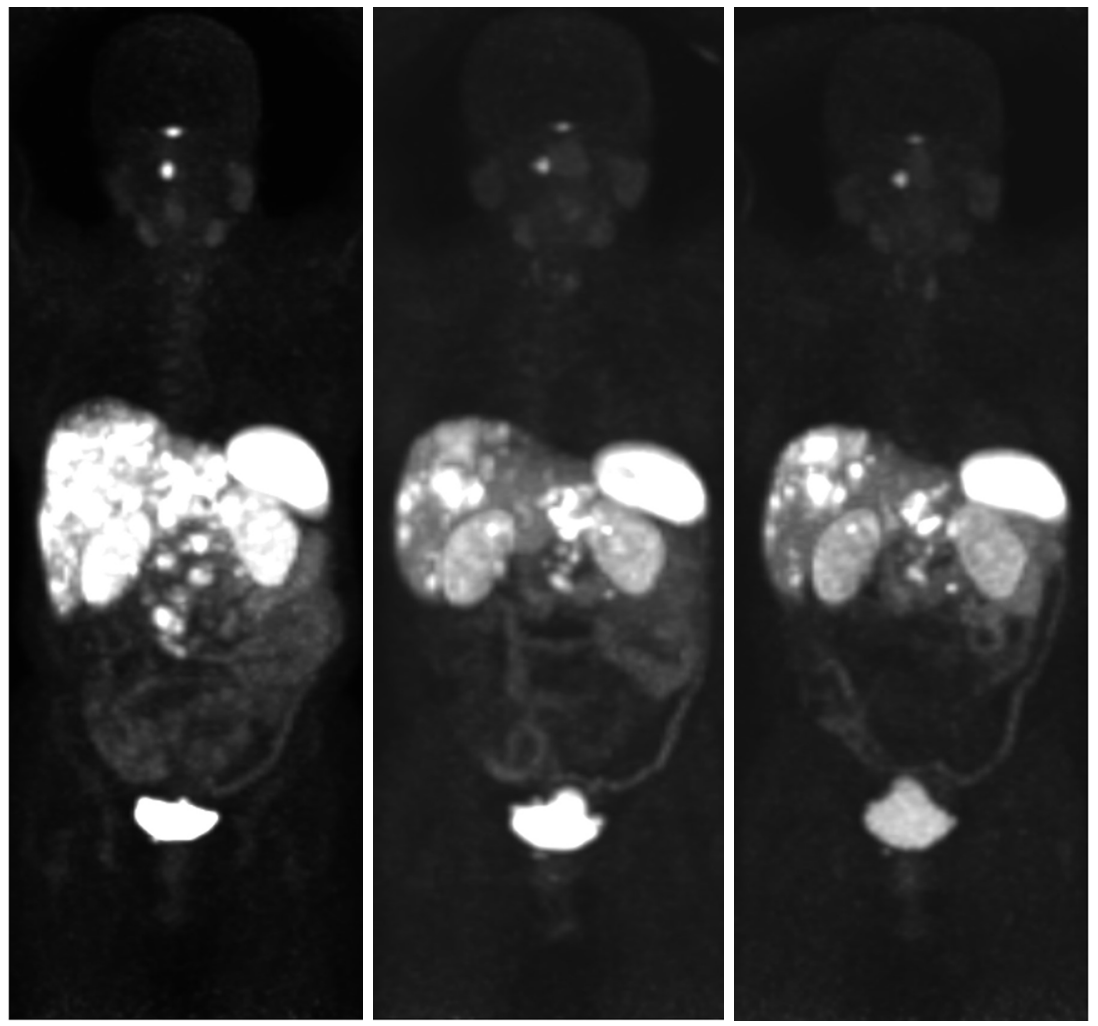

Figure 8 Serial ${ }^{68}$ Ga-DOTATATE positron emission tomography scans from July 2018 (Left), July 2019 (Middle) and November 2019 (Right) demonstrating ongoing response 14 months after initiation of peptide radionuclide receptor therapy in September 2018.

the stomach. However, there is a reported case with primary site in the duodenum in a 29-year-old (6). In our patient, clinicopathologic features were highly suggestive of SDH deficiency, but this was not identified in Nepal or India due to lack of SDH testing and erroneous lab results.

Despite showing positive IHC for cKIT and DOG1, SDH-deficient GISTs lack KIT and PDGFRA mutations, and are typically TKI resistant. To date, only a few retrospective studies have reported objective response rates of less than $20 \%$ in SDH-deficient patients receiving imatinib and sunitinib (31). The rarity and indolent nature of SDH-deficient GIST limits assessment of the relationship between genotype, response, and survival.

Both cases presented highlight the need for novel treatment approaches for metastatic SDH-deficient GIST. At the molecular level, SDH-deficient GISTs show global DNA hypermethylation as opposed to KIT/PDGRA mutated GIST (32). Although pervasive, this appears to be nonrandom and results in loss of expression of various proteins, including $\mathrm{O}^{6}$-methylguanine DNA methyltransferase (MGMT) (32). In a study comparing MGMT methylation of SDH-deficient and SDH-proficient GIST, the fraction of SDH-deficient GIST with MGMT methylation was significantly higher than SDH-proficient GIST [6/9 (67\%) vs. 6/39 (15\%), $\mathrm{P}=0.004$ ] (32). In gliomas, MGMT inactivation improves efficacy of alkylating agents. Our patient was started on temozolomide, and following an initial partial response, had stable disease 18 months after starting treatment. Currently a phase 2 single arm study is evaluating the effectiveness of temozolomide in this rare subset (33).

SDH-deficient GISTs consistently overexpress insulinlike growth factor 1 receptor (IGF-1R), and one new agent being evaluated in WT GIST is linsitinib, an oral IGF$1 \mathrm{R}$ inhibitor $(34,35)$. In a phase 2 trial with 20 WT GIST patients, PFS at 9 months was $52 \%$, however, there were no objective responses (35).

The concept of combining diagnostic and therapeutic modalities (theranostics) has been around for over 50 years, and peptide receptor scintigraphy (PRS) was first used as an imaging modality in the late 1980s (36). Peptide receptor radionuclide therapy (PRRT) was first used to treat a patient with neuroendocrine malignancy in 1992 using high 

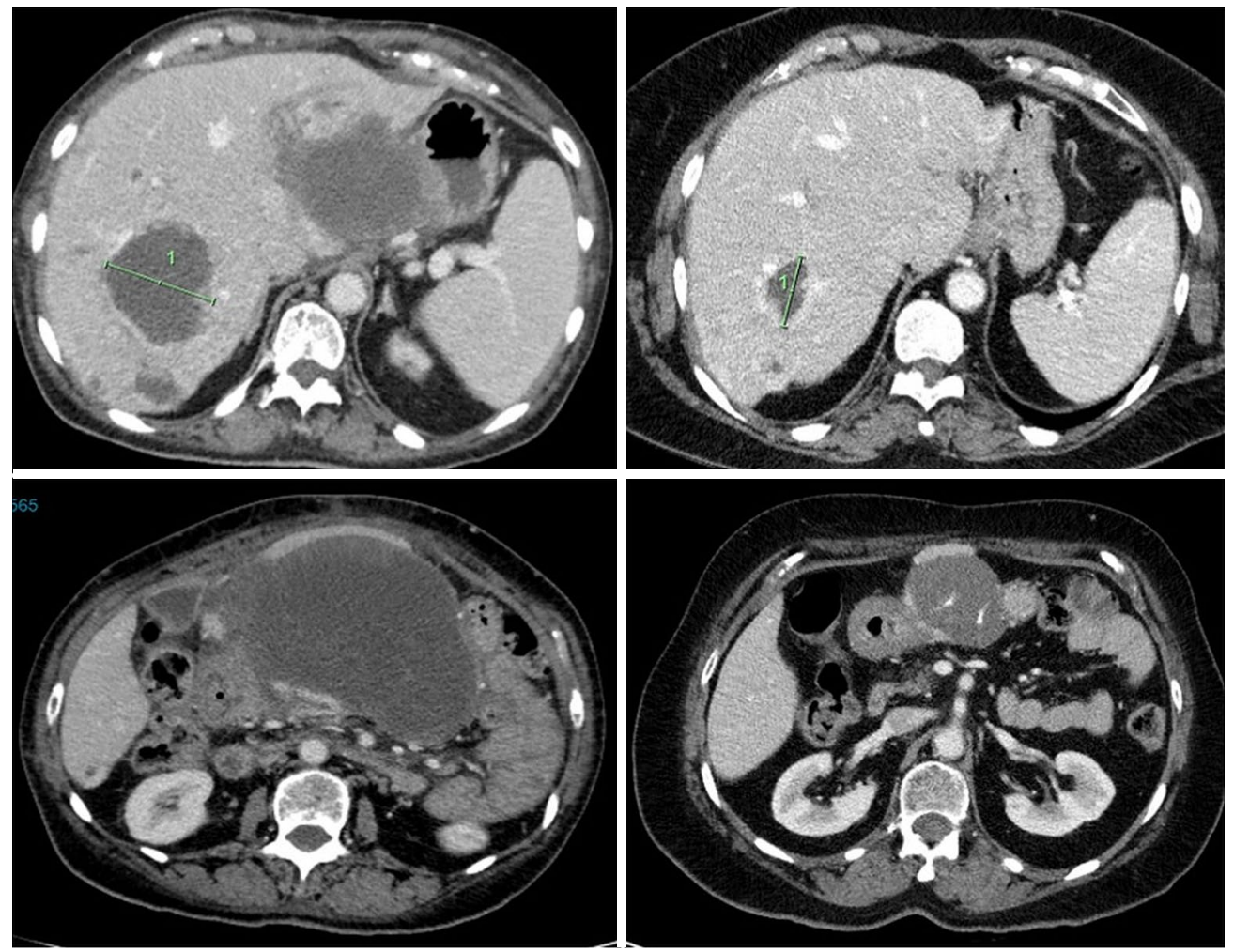

Figure 9 Computed tomography scans from July 2018 (left top \& bottom, pre-treatment) and February 2020 (right top \& bottom, 17 months post-treatment) demonstrating a deep and durable response to peptide radionuclide receptor therapy.

doses of ${ }^{111}$ Indium-pentetreotide (37). Over the subsequent two decades, PRRT has become an established therapeutic option mainly for patients with metastatic neuroendocrine tumours. In the pivotal phase III NETTER1 study, PRRT with ${ }^{177}$ lutetium-DOTA-octreotate demonstrated significant progression-free and overall survival benefit over escalated dose somatostatin therapy in patients with progressive metastatic midgut neuroendocrine malignancy (38).

The patient in case 2 was found to have avid disease on DOTATATE PET and experienced a durable partial response to ${ }^{177}$ lutetium-DOTA-octreotate PRRT after failing two lines of TKI therapy. Somatostatin receptor expression in patients with metastatic GIST has been reported, and there may be preferential expression of SSTR2 in patients with SDHdeficient disease $(20,27)$. There are currently no other case reports or prospective trials investigating PRRT in this setting, though this holds promise as a novel therapeutic approach.

\section{Conclusions}

These two interesting cases highlight several key issues with respect to the clinical management of patients with metastatic GIST. In the first case, we highlighted the significance of correlating clinical presentation with underlying biology, as well as the importance of access to genomic diagnostics as an issue in developing countries. It is clear that genotyping is paramount for GIST patients, with predictive and prognostic value, and in some cases, diagnostic value. The first case also demonstrated the potential activity of temozolomide in SDH-deficient GIST, with response and stabilization of disease lasting at least 18 months. However, given limited follow up and indolent disease course, it is difficult to infer true impact on PFS. The activity of temozolomide may be due to the higher incidence of MGMT methylation observed in SDH-deficient GIST, and is under investigation in clinical trials. In the second case, we demonstrated ${ }^{68} \mathrm{Ga}$-DOTATATE PET and PRRT as potential means to evaluate, treat and monitor carefully selected patients with metastatic GIST. Certainly, this should be further explored and validated in prospective trials. 

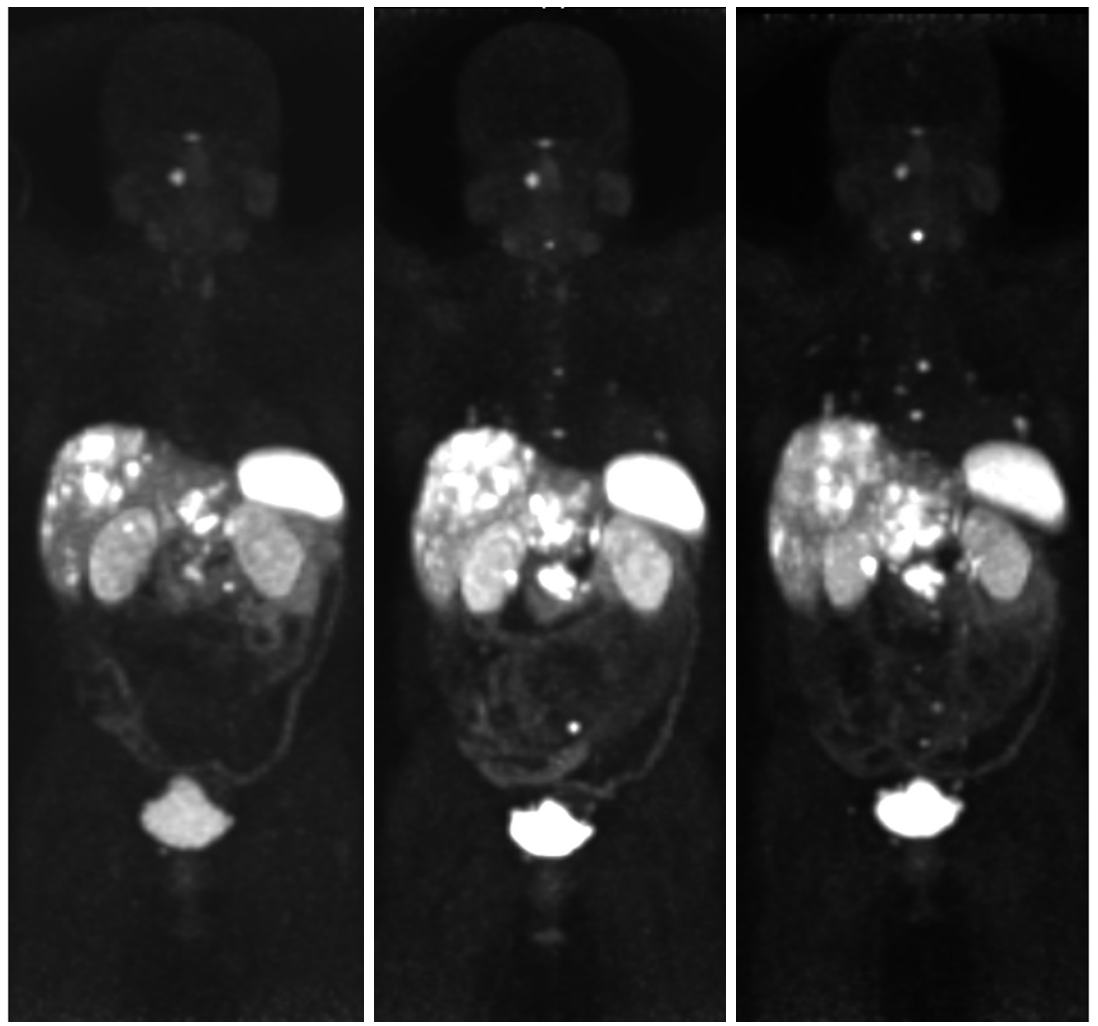

Figure 10 Serial ${ }^{68}$ Ga-DOTATATE positron emission tomography scans from November 2019 (Left), July 2020 (Middle) and December 2020 (Right) demonstrating disease progression in July 2020 (22 months after commencing peptide radionuclide receptor therapy). The patient then commenced temozolomide in September 2020. Restaging after 3 cycles in December 2020 revealed mixed response, but importantly, there was a reduction in the burden of hepatic disease.

\section{Acknowledgments}

Funding: None.

\section{Footnote}

Reporting Checklist: The authors have completed the CARE reporting checklist. Available at http://dx.doi.org/10.21037/ tcr-21-131

Conflicts of Interest: All authors have completed the ICMJE uniform disclosure form (available at http://dx.doi. org/10.21037/tcr-21-131). The authors have no conflicts of interest to declare.

Ethical Statement: The authors are accountable for all aspects of the work in ensuring that questions related to the accuracy or integrity of any part of the work are appropriately investigated and resolved. All procedures performed in studies involving human participants were in accordance with the ethical standards of the institutional and/or national research committee(s) and with the Helsinki Declaration (as revised in 2013). Written informed consent was obtained from the patients.

Open Access Statement: This is an Open Access article distributed in accordance with the Creative Commons Attribution-NonCommercial-NoDerivs 4.0 International License (CC BY-NC-ND 4.0), which permits the noncommercial replication and distribution of the article with the strict proviso that no changes or edits are made and the original work is properly cited (including links to both the formal publication through the relevant DOI and the license). See: https://creativecommons.org/licenses/by-nc-nd/4.0/.

\section{References}

1. Wang YM, Gu ML, Ji F. Succinate dehydrogenase- 
deficient gastrointestinal stromal tumours. World J

Gastroenterol 2015;21:2303-14.

2. Gill AJ, Chou A, Vilain R, et al. Immunohistochemistry for SDHB divides Gastrointestinal Stromal Tumors (GISTs) into two distinct types. Am J Surg Pathol 2010;34:636-44.

3. Gill AJ. Succinate dehydrogenase (SDH) and mitochondrial driven neoplasia. Pathology 2012;44:285-92.

4. Gill AJ. Succinate dehydrogenase (SDH)-deficient neoplasia. Histopathology 2018;72:106-16.

5. Gill AJ, Chou A, Vilain RE, et al. "Pediatric type" Gastrointestinal stromal tumors are SDHB negative ("type 2") GISTs. Am J Surg Pathol 2011;35:1245-7.

6. Elston MS, Sehgal S, Dray M, et al. A Duodenal SDHDeficient Gastrointestinal Stromal Tumor in a Patient with a Germline SDHB Mutation. J Clin Endocrinol Metab 2017;102:1447-50.

7. Zhang L, Smyrk TC, Young WF, et al. Gastric Stromal Tumors in Carney Triad Are Different Clinically, Pathologically, and Behaviorally From Sporadic Gastric Gastrointestinal Stromal Tumors: Findings in 104 Cases. Am J Surg Pathol 2010;34:53-64.

8. Killian JK, Kim SY, Miettinen M, et al. Succinate dehydrogenase mutation underlies global epigenomic divergence in gastrointestinal stromal tumor. Cancer Discov 2013;3:648-57.

9. Dwight T, Benn DE, Clarkson A, et al. Loss of SDHA expression identifies SDHA mutations in succinate dehydrogenase deficient gastrointestinal stromal tumors. Am J Surg Pathol 2013;37:226-33.

10. Joensuu H, Vehtari A, Riihimaki J, et al. Risk of recurrence of gastrointestinal stromal tumour after surgery: an analysis of pooled population-based cohorts. Lancet Oncol 2012;13:265-74.

11. Patrikidou A, Domont J, Chabaud S, et al. Long-term outcomes of molecular subgroups of GIST patients treated with standard-dose imatinib in the BFR14 trial of the French Sarcoma Group. Eur J Cancer 2016;52:173-80.

12. Demetri GD, von Mehren M, Blanke CD, et al. Efficacy and Safety of Imatinib Mesylate in Advanced Gastrointestinal Stromal Tumors. N Engl J Med 2002;347:472-80.

13. Casili PG, Zalcberg J, Le Cesne A, et al. Ten-Year Progression-Free and Overall Survival in Patients With Unresectable or Metastatic GI Stromal Tumours: LongTerm Analysis of the European Organisation for Research and Treatment of Cancer, Italian Sarcoma Group, and Australasian Gastrointestinal Trials Group Intergroup
Phase III Randomized Trial on Imatinib at Two Dose Levels. J Clin Oncol 2017;25:1713-20.

14. Demetri GD, van Oosterom AT, Garrett CR, et al. Efficacy and safety of sunitinib in patients with advanced gastrointestinal stromal tumour after failure of imatinib: a randomised controlled trial. Lancet 2006;368:1329-38.

15. Demetri GD, Reichardt P, Kang YK, et al. Efficacy and safety of regorafenib for advanced gastrointestinal stromal tumours after failure of imatinib and sunitinib: an international, multicentre, prospective, randomised, placebo-controlled phase 3 trial (GRID). Lancet 2013;381:295-302.

16. Shah K, Chan K, Ko Y. A systematic review and network meta-analysis of post-imatinib therapy in advanced gastrointestinal stromal tumour. Curr Oncol 2017;24:e531-9.

17. Vallilas C, Sarantis P, Kyriazoglou A, et al. Gastrointestinal Stromal Tumors (GISTs): Novel Therapeutic Strategies with Immunotherapy and Small Molecules. Int J Mol Sci 2021;22:493.

18. Serrano C, Leal A, Kuang Y, et al. Phase I Study of Rapid Alternation of Sunitinib and Regorafenib for the Treatment of Tyrosine Kinase Inhibitor Refractory Gastrointestinal Stromal Tumors. Clin Cancer Res 2019;25:7287-93.

19. Ray-Coquard I, Cassier P, Galy I, et al. Combination therapy for gastrointestinal stromal tumours: evidence from recent clinical trials. Clin Invest 2011;1:825-36.

20. Arne G, Nilsson B, Dalmo J, et al. Gastrointestinal Stromal Tumours (GISTs) express somatostatin receptors and bind radiolabeled somatostatin analogues. Acta Oncol 2013;52:783-92.

21. Zhao WY, Zhuang C, Xu J, et al. Somatostatin receptors in gastrointestinal stromal tumours: new prognostic biomarker and potential therapeutic strategy. Am J Transl Res 2014;6:831-40.

22. Loaiza-Bonilla A, Bonilla-Reyes PA. Somatostatin Receptor Avidity in Gastrointestinal Stromal Tumours: Theranostic Implications of Gallium-68 Scan and Eligibility for Peptide Receptor Radionuclide Therapy. Cureus 2017;9:e1710.

23. Paulmichl A, Summer D, Manzl C, et al. Targeting Gastrointestinal Stromal Tumour with 68Ga-Labeled Peptides: An In Vitro Study on Gastrointestinal Stromal Tumour Cell Lines. Cancer Biother Radiopharm 2016;31:302-10.

24. Suresh Babu MC, Chaudhuri T, Babu KG, et al. Metastatic gastrointestinal stromal tumor: A regional 
cancer center experience of 44 cases. South Asian J

Cancer. 2017;6:118-21.

25. Iqbal N, Sharma A, Shukla NK, et al. Advanced gastrointestinal stromal tumors: 10-years experience from a tertiary care centre. Trop Gastroenterol 2015;36:168-73.

26. Darling HS, Rastogi S. Rare cancers in India: A road less travelled. Indian J Cancer 2020;57:139.

27. Elston MS, Meyer-Rochow GY, Conaglen HM, et al. Increased SSTR2a and SSTR3 expression in succinate dehydrogenase-deficient pheochromocytomas and paragangliomas. Hum Pathol 2015;46:390-6.

28. Körner M, Waser B, Schonbrunn A, et al. Somatostatin receptor subtype $2 \mathrm{~A}$ immunohistochemistry using a new monoclonal antibody selects tumors suitable for in vivo somatostatin receptor targeting. Am J Surg Pathol 2012;36:242-52.

29. Pantaleo MA, Lolli C, Nannini M, et al. Good survival outcome of metastatic SDH-deficient gastrointestinal stromal tumors harboring SDHA mutations. Genet Med 2015;17:391-5.

30. Miettinen M, Wang Z-F, Sarlomo-Rikala M, et al. Succinate Dehydrogenase deficient GISTS - A Clinicopathologic, Immunohistochemical, and Molecular Genetic Study of 66 Gastric GISTS with Predilection to Young Age. Am J Surg Pathol 2011;35:1712-21.

31. Call J, Wang Y, Rothschild, et al. Treatment Responses in SDH-Deficient GIST. Patient-reported Treatment Responses in Known/Likely SDH-deficient GISTS: An analysis of The Life Raft Group Observational Registry [Internet]. Wayne, New Jersey: The Life Raft Group; August 2019 [cited 31 Mar 2021]. Available online: https:// adobeindd.com/view/publications/a35275fd-793a-4173b6d3-a3c304f381e0/cnvl/publication-web-resources/pdf/ LRG_Science_August_2019.pdf

32. Ricci R, Martini M, Ravegnini G, et al. Preferential MGMT methylation could predispose a subset of KIT/ PDGFRA-WT GISTs, including SDH-deficient ones, to respond to alkylating agents. Clin Epigenetics 2019;11:2.

33. ClinicalTrials.gov. An Open-Label, Phase 2 Efficacy Study of Temozolomide (TMZ) In Advanced Succinate Dehydrogenase (SDH)-Mutant/Deficient Gastrointestinal Stromal Tumor (GIST). Identifier: NCT03556384. Available online: https://clinicaltrials.gov/ct2/show/ NCT03556384

34. Chou A, Chen J, Clarkson A, et al. Succinate Dehydrogenase Deficient GISTs Are Characterized By IGF1R Overexpression. Mod Pathol 2012;25:1307-13.

35. von Mehren M, George S, Heinrich MC, et al. Linsitinib (OSI-906) for the treatment of Adult and Pediatric Wild Type Gastrointestinal Stromal Tumors, a SARC Phase II study. Clin Cancer Res 2020;26:1837-45.

36. Krenning EP, Bakker WH, Breeman WA, et al. Localisation of endocrine-related tumours with radioiodinated analogue of somatostatin. Lancet 1989;1:242-4.

37. Krenning EP, Kooij PP, Bakker WH, et al. Radiotherapy with a radiolabeled somatostatin analogue, [111In-DTPAD-Phe1]-octreotide. A case history. Ann N Y Acad Sci 1994;733:496-506.

38. Strosberg J, El-Haddad G, Wolin E, et al. Phase 3 Trial o Lu-Dotatate for Midgut Neuroendocrine Tumours. N Engl J Med 2017;376:125-35.
Cite this article as: De Silva M, Rastogi S, Chan D, Angel C, Prall O, Gill A, Guminski A. Succinate dehydrogenase-deficient gastrointestinal stromal tumor: from diagnostic dilemma to novel personalised therapy in 2 case reports. Transl Cancer Res 2021;10(7):3588-3599. doi: 10.21037/tcr-21-131 